\title{
Motion Planning Of An Autonomous Mobile Robot Using ARTIFICIAL NEURAL NETWORK
}

\author{
G. N. Tripathi ${ }^{1}$ and V.Rihani ${ }^{2}$ \\ ${ }^{1}$ Mody Institute of Technology and Science, Lakshamangarh, Sikar, Rajasthan \\ gyanendra2004@gmail.com \\ ${ }^{2}$ E\&EC Department, PEC University of Technology, Chandigarh, India \\ v.rihani@yahoo.com
}

\begin{abstract}
The paper presents the electronic design and motion planning of a robot based on decision making regarding its straight motion and precise turn using Artificial Neural Network (ANN). The ANN helps in learning of robot so that it performs motion autonomously. The weights calculated are implemented in microcontroller. The performance has been tested to be excellent.
\end{abstract}

\section{KEYWORDS}

Autonomous Robot, Artificial Neural Network (ANN), Motion Planning, Mobile Robot, Robot Intelligence and Machine Learning.

\section{INTRODUCTION}

ANN provides high speed data processing capability of learning [1]. The use of ANN in robotics for its kinematics, dynamics, and path planning and motion control has changed the definition of the robot [2].

As per Webster a Robot is:

"An automatic device that performs functions normally ascribed to humans or a machine in the form of a human".

A Mobile Robot is a robot system that is capable of moving within an environment or terrain where it performs the tasks. Using an ANN technique, adequate human like decision making quality can be built in a robot. This requires the analysis of environment to resolve complexity of motion and ensured that the robot is mechanically characterized to perform motion, with required degree of freedom. Then electronic has to support the motor used, hence to control the motion.

\section{Present level}

Mobile robot motion planning and path planning is one of the most apparent field of application of ANN. If a robot encounters an obstacle, the arm attempts to avoid the obstacle [3]. The image processing can ensure robot obstacle avoidance and path planning in a two dimensional work space of the robot [4]. A recurrent net has been used in prediction of the motion of an object in a robot path navigation system [5]. A new learning algorithm for learning of self-organizing neural networks is proposed to recognize the traffic signs for navigation of a mobile robot in an outdoor

Natarajan Meghanathan, et al. (Eds): SIPM, FCST, ITCA, WSE, ACSIT, CS \& IT 06, pp. 367-373, 2012. (C) CS \& IT-CSCP 2012 
environment [6]. Image matching is used to make decision for object [7]. An adaptive neural network can accept visual signals as inputs directly from visual sensors for the spatial information [8]. ANNs can plan motion of several mobile robots ensuring collision avoidance [9]. Fuzzy modelling of the real robot's environment using Hopfield neural network has been used [10]. ANN base motion planer can respond to changing real time situation [11]. Latency effect in a closed loop system can be reduced for motion prediction [12]. To design the vehicle controller behavioral cloning machine learning algorithm and neural network algorithms can also be used [13]. Improvements have been made using fuzzy logic based path planning algorithm and are more effective. [14]. Convergence of a Neural Network has been improved using Q-Learning (NCQL) algorithm [15]. Extended Back Propagation Algorithm predicts moving obstacles for obstacle avoidance [16]. Designing a robot and planning its motion is always a new task in a new environment.

\section{THE ELECTRONICS}

The 2D motion of designed robot is performed using two motors, one for forward drive and another for rotation of steering to turn the vehicle direction using microcontroller is shown in the figure 3.1.

PIC16f877a has been used for the design of control circuit which has computational speed, memory (8K) and on chip ADC.

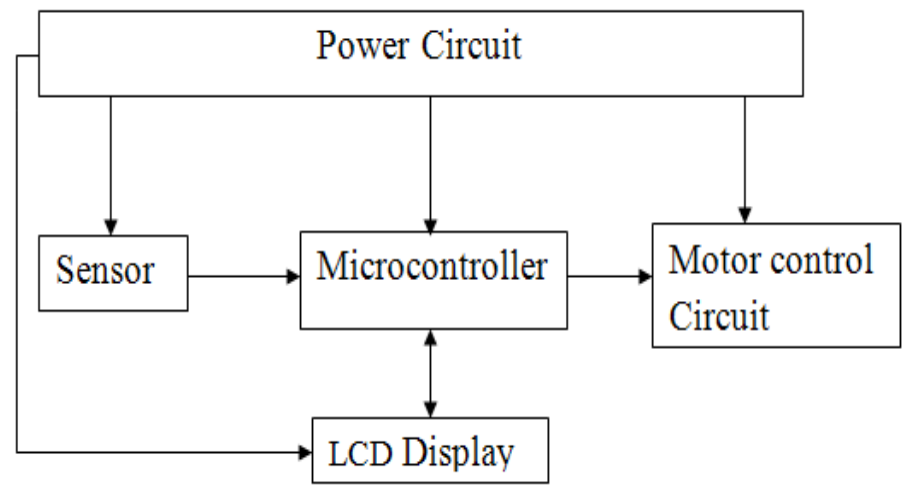

Figure 3.1 block diagram of electronic circuit

\subsection{Sensor}

Distance measuring sensor GP2Y0A21YK0F, having a PSD (position sensitive detector), IRED (infrared emitting diode) and signal processing circuit, has been used. The sensor is capable of meeting the requirements of range of distance measurement, low power consumption and package size. Output of the sensor is voltage analogous to the distance of an object.

\section{ARTIFICIAL NEURAL NETWORK IMPLEMENTATION}

Feed forward neural network is used to make decision regarding the motion of the robot.

\subsection{The Environment}

The walls of the environment are made up of Medium Density Fiber (MDF) board and wood strips. Walls are attached with each other at angle of 90 degree. 


\subsection{Application of ANN for decision making}

The mobile robot designed has an Infrared sensor (a distance meter) and can scan the space in front from -90 to +90 degrees. The sensing has been made at $-90,-45,0,+45,+90$ degree angles. The signal of the sensor at these angles is input to microcontroller sequentially. The ADC of microcontroller gives ' 8 ' bit digital output corresponding to the five different signals. The output of ADC is converted to binary using the threshold function.

The threshold ('th') is chosen based on the distance and corresponding ADC value sensed. Using this, ANN decides straight, left and right motion of the robot.

\subsection{The analysis of path of environment}

The top view of the path is shown here and the scanning condition for straight, left and right motion is also shown in fig.

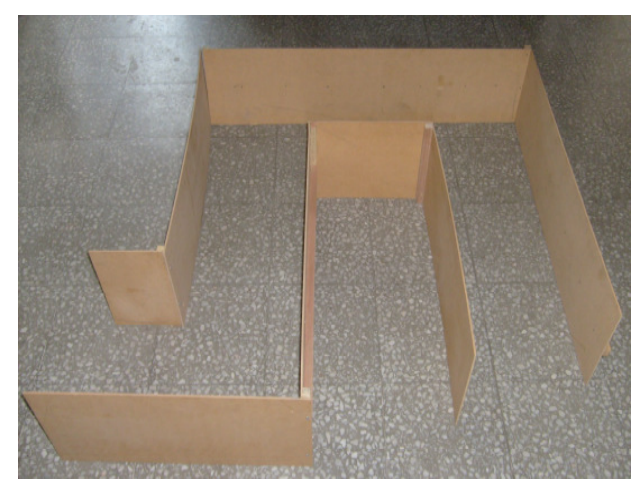

Figure-4.1(a) Real image of environment
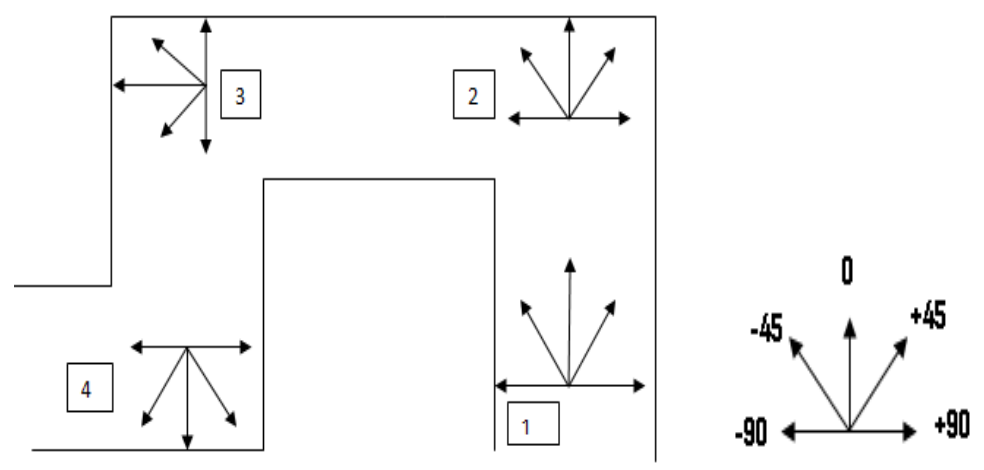

Figure-4.1(b) Top view of walls and scanning position

The point 1, 2, 3, 4 represent front area scanning point, same as shown in figure 4.1, with a changing front space.

Input to five input layer neurons is the output of the threshold function corresponding to the ADC value sensed at angles $-90,-45,0,+45,+90$. These five inputs are represented by variables $X 1$, $\mathrm{X} 2, \mathrm{X} 3, \mathrm{X} 4, \mathrm{X} 5$ corresponding to angles $-90,-45,0,+45,+90$ as in Figure 4.2.

1. At this position the threshold Function output for ' $\mathrm{X} 1$ ' and ' $\mathrm{X} 5$ ' will be ' 1 ' and it will represent the presence of obstacle on both left and right side of vehicle, 2. and 3. are the position where threshold function output for 'X3' and 'X5' will be ' 1 ' that will represent the presence of obstacle on front and right side of vehicle, 4. at this point the threshold function Output for 'X1' and ' $\mathrm{X} 3$ ' will be ' 1 ' that will represent the presence of obstacle on front and left side of vehicle. The stopping input condition for the vehicle is given when all inputs are ' 1 '. 
Table-4.1 Input combination and corresponding outputs of ANN simulation

\begin{tabular}{|c|c|c|c|c|c|c|}
\hline & $\mathrm{X1}$ & $\mathrm{X} 2$ & $\mathrm{X3}$ & $\mathrm{X4}$ & $\mathrm{X5}$ & Output \\
\hline \multirow{5}{*}{1.} & 1 & 1 & 0 & 1 & 1 & straight \\
\hline & 1 & 0 & 0 & 1 & 1 & Straight \\
\hline & 1 & 1 & 0 & 0 & 1 & Straight \\
\hline & 1 & 0 & 0 & 0 & 1 & Straight \\
\hline & 0 & 0 & 0 & 0 & 0 & straight \\
\hline \multirow{3}{*}{$\begin{array}{l}2 . \\
\&\end{array}$} & 0 & 1 & 1 & 1 & 1 & Left \\
\hline & 0 & 0 & 1 & 1 & 1 & Left \\
\hline & 0 & 1 & 1 & 0 & 1 & Left \\
\hline 3. & 0 & 0 & 1 & 0 & 1 & Left \\
\hline \multirow{5}{*}{4.} & 1 & 1 & 1 & 1 & 0 & Right \\
\hline & 1 & 1 & 1 & 0 & 0 & Right \\
\hline & 1 & 0 & 1 & 1 & 0 & Right \\
\hline & 1 & 0 & 1 & 0 & 0 & Right \\
\hline & 1 & 1 & 1 & 1 & 1 & No Movement \\
\hline
\end{tabular}

\subsection{Training ANN}

Easy NN-plus has been used. It is trained using actual data and generates weights of connections for optimized condition using Back Propagation Algorithm and with 'tanh' sigmoid threshold function.

\section{Steps used in Easy NN-plus}

Step1.Supervised learning is used. For this both inputs and outputs are defined according to that. Data for Input to the Input Neurons and Output from Output Neurons are prepared as a first step.

Step2.The network is trained and the generated weight file contains the value of associations of interconnections and bias value.

\subsection{The value of weights are given as}

Weights from input layer to hidden Wij and bias Bwj.

Wij= Weight of connection from ith input layer neuron to jth hidden layer neuron.

$\mathrm{Bwj}=$ Biasing weight for $\mathrm{jth}$ hidden layer neuron.

Weights from Hidden layer to output layer Ujk and bias Buk

$\mathrm{Ujk}=$ weight of connection from jth hidden layer neuron to kth output layer neuron.

Buk= bias value for output layer neuron.

\section{TeSTING}

Various tests are performed to carry out the measurement for achieving the desired accuracy levels. This includes the decision making.

\subsection{Vehicle forward motion testing}

This is carried out to to have practical observation that how many steps of stepper motor are needed to move a particular distance. A four ' 4 ' bit combination has been used in a loop to drive the stepper motor for multiple steps. So, number of steps $=(\text { number of loop count })^{*} 4$ 
Nested loops are used for driving the motor for steps more than '1024' (number of steps $=256^{* 4}$ ). The inner loop is kept at ' 50 ' count and outer loop count was varied to observe counts it takes to move 6 inches. Due to nonlinearity of sensors, the distance from obstacle was divided in to 6 inches slot as it was observed after testing sensor that for each 6 inch slot sensor out put is linear. Experimentally total number of steps $=$ (number of loop count for outer loop)*(number of loop count for inner loop) $* 4 \quad=7 * 50 * 4$

$$
=1400
$$

\subsection{Calibration and testing of sensor}

Sensor testing is performed to get the relation of the digital output (digital output given by the controller corresponding to the sensors output voltage) to the distance from the wall. Digital value is proportional to the output voltage of sensor hence; it is proportional strength of infrared signal reflected from obstacle.

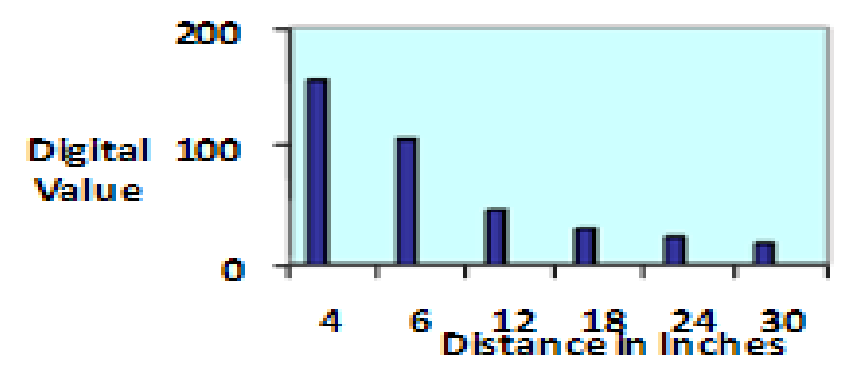

Figure-5.1 Graph chart for digital value of Sensor data to distance

\subsection{Steering test for scanning of front area}

For turning the front wheel, following steps were taken and data stored in controller. Initially it is assumed that the sensor is at ' 0 ' degree position.

1. The sensor signal, at ' 0 ' degree, is acquired and displayed to LCD

2. Sensor is turned to -45 degree, sensor signal acquired and displayed.

3. Sensor is turned to -90 degree, sensor signal acquired and displayed.

4. Sensor is turned to ' 0 ' degree position by rotating it +90 degree, sensor signal acquired and displayed again.

5. Sensor is turned to +45 degree, sensor signal acquired and displayed.

6. Sensor is turned to +90 degree, sensor signal acquired and displayed.

7. Sensor is turned to ' 0 ' degree position by rotating it -90 degree.

\subsection{Left turn and right turn testing}

The left and right turn test is done to check how many forward motion steps are required by the vehicle to complete the turning motion. It is observed that when -45 degree and +45 degree rotation is used for left and right turn the curvature of the vehicle motion was long and the body of vehicle reaches close to the wall of environment. Therefore, to avoid collision -60 degree and +60 degree rotation is required for left turn and right turn.

The steps performed in program:

1. Rotate the wheel at -60 for left or +60 for right motion

2. Stepper Motor for forward motion is started to move forward

3. Loop count for the forward motion is displayed 
4. After completion of desired turn wheel is again rotated by +60 or -60 to bring the wheel to ' 0 ' position

The required forward motion steps for complete turn are calculated as:

Total number of steps $=($ number of loop count for outer loop)* (number of loop count for inner loop) $* 4$

\subsection{Simulation test for artificial neural network}

Output of ANN is used in Flowcode software. Input to the network is the output of threshold function applied to digital value of ADC, on scanning front area. The output of the network is the decision of either going straight or making a turn to left or right based on.

Threshold value 'th' $=95$ has been used which is Digital value of ADC corresponding to vehicle position with respect to wall. It is the position from which vehicle needs to turn left or right, to avoid collision.

The required ANN output was achieved using ' 0.8 ' as threshold for activation function.

\subsection{Testing of autonomous vehicle}

ANN performed well to make decision for the motion of vehicle in real environment as it was noted during simulation table 4.1 .

\section{Conclusions}

The designed robot is capable of moving autonomously in the environment for which it is trained. It senses the wall as obstacle and makes decision to go straight, right or left according to environment. With the use of Artificial Neural Network, self decision making by robot is performed. This robot is designed for a real time implementation of ANN for intelligent system. Use of the ANN in system improves performance.

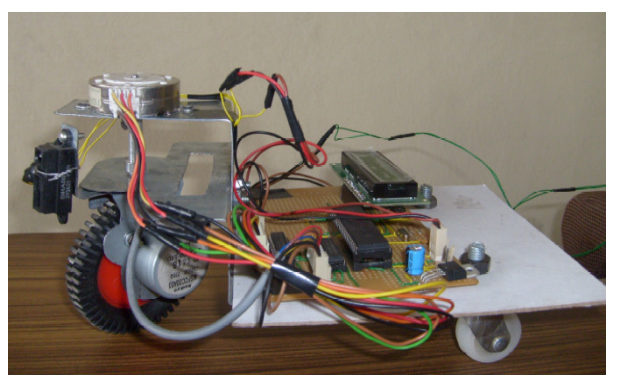

Image of Robot

\section{REFERENCES}

[1] Bishop, C.M. (1994, June). Neural networks and their applications. Review of Scientific Instruments, Vol.65 (6), 1803-1832.

[2] Tzafestas, S.G. (Jul1995 ). Neural networks in robotics: state of the art. Industrial Electronics, 1995. ISIE '95., Proceedings of the IEEE International Symposium, 1, (pp 12 - 20) .

[3] Onema, J.P., Schreur, B., Mclauchlan, R.A., \& Ashtijou, M. (Jul 1991 ). A Motion planning strategy and its neural network implementation for an articulated two-link arm with scissor hand system . Neural Networks, 1991., IJCNN-91-Seattle International Joint Conference , 2, (pp 1005 ).

[4] Kozakiewicz, C., Ejiri, M. (Nov 1991 ). Neural network approach to path planning for two dimensional robot motion. Intelligent Robots and Systems '91. 'Intelligence for Mechanical Systems, Proceedings IROS '91. IEEE/RSJ International Workshop , 2, (pp 818-823). 
[5] Meng, H., Picton, P.D. (May 1993 ). A Neural network motion predictor. Artificial Neural Networks, 1993., Third International Conference, (pp 177-181).

[6] Luo, R.C., Potlapalli, H. (Jul 1994 ). Landmark recognition using projection learning for mobile robot navigation. Neural Networks, 1994. IEEE World Congress on Computational Intelligence., 1994 IEEE International Conference , 4, (pp 2703-2708).

[7] Kai-Tai Song Jhy-Min Chang (Aug 1996 ). Experimental study on robot visual tracking using a neural controller. Industrial Electronics, Control, and Instrumentation, 1996., Proceedings of the 1996 IEEE IECON 22nd International Conference, 3, (pp 1850-1855).

[8] Shibata, K., Okabe, Y. (Jun 1997 ). Reinforcement learning when visual sensory signals are directly given as inputs. Neural Networks, 1997., International Conference, 3, (pp 1716-1720).

[9] Kolushev, F.A., Bogdanov, A.A. (Jul 1999 ). Neural algorithms of path planning for mobile robots in transport systems. Neural Networks, 1999. IJCNN '99. International Joint Conference, 6, (pp 43814386).

[10] Sadati, N., Taheri, J. (2002). Solving robot motion planning problem using hopfield neural network in a fuzzified environment. Fuzzy Systems, 2002. FUZZ-IEEE'02. Proceedings of the 2002 IEEE International Conference, 2, (pp 1144-1149).

[11] Erickson, D. (Oct. 2003). Non-learning artificial neural network approach to motion planning for the pioneer robot. Intelligent Robots and Systems, 2003. (IROS 2003). Proceedings. 2003 IEEE/RSJ International Conference, $1,(\mathrm{pp} 112-117)$.

[12] Yu Sheng Yonghai Wu. (Nov. 2005 ). Motion prediction in a high-speed, dynamic environment. Tools with Artificial Intelligence, 2005. ICTAI 05. 17th IEEE International Conference, (pp 705).

[13] Kulic, R., Vukic, Z. (Nov. 2006 ). Autonomous vehicle obstacle avoiding and goal position reaching by behavioral cloning. IEEE Industrial Electronics, IECON 2006 - 32nd Annual Conference, (pp 3939-3944).

[14] Zhenwen Su Bi Zeng Guangchang Liu Feng Ye Minglin Xu. (June 2007). Application of fuzzy neural network in parameter optimization of mobile robot path planning using potential field. Industrial Electronics, 2007. ISIE 2007. IEEE International Symposium, (pp 2125-2128).

[15] Zheng Qin Gu, J. (Aug. 2009). Neural q-learning in motion planning for mobile robot. Automation and Logistics, 2009. ICAL '09. IEEE International Conference, (pp 1024-1028).

[16] Engedy, I., Horvath, G. (Aug. 2009 ). Artificial neural network based mobile robot navigation. Intelligent Signal Processing, 2009. WISP 2009. IEEE International Symposium, (pp 241-246).

G. N. Tripathi Received B.E. Degree from Birla Institute of Applied Sciences, Bhimatal, Uttarakhand and M.E. Degree from PEC University of Technology, Chandigarh. He is working as Assistant Professor, ECE, Mody Institute of Technology, Lakshamangarh, Rajasthan. His research interests are Microcontroller based system design, embedded design for robots and intelligent systems, Neural Network.

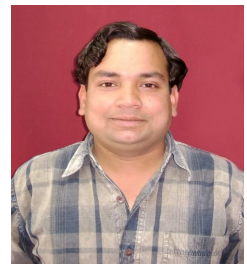

Prof. V. Rihani worked as faculty and HOD, E\&EC department, PEC University of Technology (formerly Punjab Engineering College), Chandigarh. His research interests are Microprocessor and Controller, Computer Architecture, Neural Networks, Microwaves and Antennas.

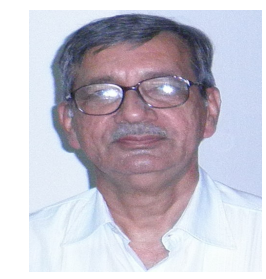

